\title{
Editoríal.
}

\section{CEREBRAL LOCALIZATION OF FUNCTION.}

THE conceptions of the physiology of the brain with regard t 1 localization of function have repeatedly swung from one extremb to the other. The older philosophic idea of the brain as the rathe indefinite seat of a general function was challenged in the early years of the nineteenth century by Gall, Spurzheim and Broussais, who attempte里 to place complex and ill-defined functions in small specific parts of the cerebral hemisphere. The extravagances of the pscudo-science of phrenology erceted upon this carly and crude theory of cerebral localizao $\overrightarrow{0}$ tion soon led to reaction; and Flourens, largely as a result of his experi ments on pigeons, reverted to the conception of absence of specifio localization of function. But this position was again shaken by this clinical observations of Broca, which seemed to indicate an intimate connection of the posterior part of the human third left frontal con volution with the function of speech; and the position finally yielder $\frac{\square}{\dot{ }}$ for a time with the discovery of the excitability of the central cores or volution by Fritsch and Hitzig in $\mathbf{1 8 7 0}$.

Electrical stimulation of the central convolution was found to evoke movements of the crossed limbs and trunk. Against this apparent confirmation by the stimulating electrode of the theory of cerebraf localization of function there are, however, to be placed the results $0 \Phi$ ablation experiments; for almost complete (if not actually complet $\$$ recovery of function may succeed initial paralysis when the lesion has o destroyed the greater part or the whole of the Rolandic motor arean These and similar results have swayed thought in one direction or the other. Some investigators have been attracted by far-reaching theorie $\vec{e}$ of spccific localization; others have been led rather to doubt the validit or of such theories; and much time has been wasted in controversy. The experimental facts which we have before us undoubtedly point, however, to some form of localization of function in the brain. Destruction of both occipital lobes deprives an animal of all its general reactions to visual stimuli. Stimuli applied to different parts of the Rolandic area cvoke different specific movements of the crossed side of the body. Stimuli applied to the cortex of the occipital pole, to that of the temporal lobe, or to that of the prefrontal lobe, give combined movements of 3 
head and eyes. But ablation of the motor area does not give permanent paralysis of the movements which may be evoked from it by electrical stimulation-even when these movements are as complex as the unlocking of a door and the turning of its handle in the case of an anthropoid ape.

The effects of electrical stimulation of the Rolandic area are, as it were, a prime foundation of the theory of cerebral localization. In a highly organized brain, such as that of an anthropoid ape, an electrica? stimulus applied to the appropriate point on the ascending frontal convolution evokes so finely differentiated a movement as extension of the crossed index finger. It is hardly too much to say that by selecting the appropriate point on this convolution any single movement which may be evoked as a voluntary act in the daily behaviour of an animalo may be clicited by the electrical stimulus. Even certain combinations of movements may be evoked if strong stimuli, or long continued stimuli,o are used. The movement then commences with that occasioned by minimal stimulation of the selected point, and extends from it to the neighbouring parts of the body in the anatomical sense. Primary movement of the index finger is thus followed by movement of the whole hand, wrist, elbow, shoulder, in that order. This " march" of movementro may be so exaggerated as to become ' cortical epilepsy' and to spreadN్ to other limbs. It is, however, to be noted that such combinations of movement are 'anatomical ' rather than 'physiological.' The order of movements in a normal series of everyday life does not march in regular: sequence from joint to joint. Stimulation of the motor cortex, on the other hand, never evokes such a pattern of movement as that exhibited, for instance, in the gymnastic anties of a monkey. Again, it should be noted that in normal reflex movement of a limb not only does its crossed neighbour take part but the remaining pairs of limbs join in the move ? ment. Movement at the lowest level appears as a widespread pattern in which all segments of the body play their part. It is a peculiarity of the movements evoked by cortical stimulation that the reaction itselfo may be perfect as far as its own limb is concerned (contraction of ond set of muscles in that limb and inhibitory relaxation of the antagonist in the same limb) but completely unaccompanied by movement of the crossed neighbouring limb. It would therefore appear that there is a finely differentiated mechanism in the motor cortex which does not set into action spinal centres in the same manner as do the in-going nerveimpulses carried by the sensory nerves of a limb, but is able in some way to pick out and set into action small portions, as it were, of the simple reflex movements. The analogy according to which the excitable points of the motor cortex are likened to the keys of a pianoforte is a crude but legitimate one. Perhaps our greatest problem is to determine in what manner the tune itself is made. 
If we liken the motor cortex to a pianoforte, the tune might be played by an internal agency comparable to the mechanism of a selfplaying piano, or by an outside agency comparable to the pianist. In the former case there would be within the motor area itself an association mechanism for setting into activity widely different motor points at one and the same time, and for giving successions of such 'chords.' The fact that very complex series of movements (such as those exhibited by the ape in opening a locked door with a key) may recover after destruction of the corresponding Rolandic area, seems, however, to negative this possibility. If the integrating agency is external to the motor area itself, we must look to other parts of the brain to provide ' association' mechanisms.

Any far-reaching theory of minute cerebral localization of function naturally requires an integrating mechanism if the fects of everyday life are to be explained. Even in the case of activit eses reprented in a single area (such as the motor area) an integrating mechanism appears to be necessary. The necessity becomes greater whgre an integration of activities in different cortical areas has to be ex glained. As is well known, Flechsig postulated three chief associative aentres on embryological grounds - one in front of the motor area pp.

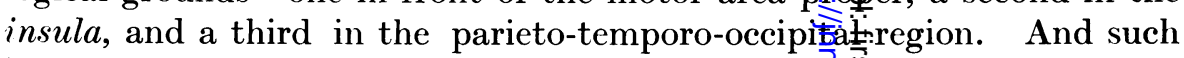
investigators as Hitzig, Bianchi, Ferrier, and many others have considered that higher associative functions are loca tis. areas of the cerebral cortex. But recent work thi

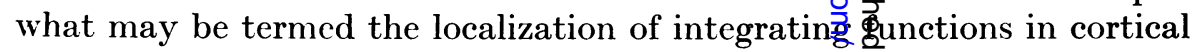
areas. The work of such American workers as Fronger and Lashley, who have made use of learnt tricks taught with the aid tion box, may be instanced. Monkeys and cats may completely lose the trick after extirpation of the frontal lobes if very firmly established; but if the trick has beefơ firmly established it may persist after the ablation. In any case thew Fick may be learnt again after the lesion has been made, and a conclusis in that the fror

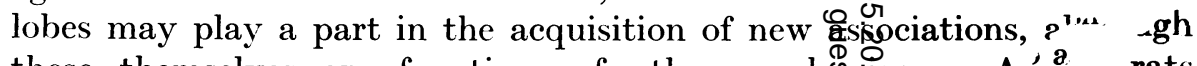
these themselves are functions of other mechenusms. A, a, rats trained to pass through a maze or to open a doकुroby $r$ " "ig over a tilting board may preserve the habit after destringetion "it the frontal lobes or of any part of the temporal or parietal $\mathbb{R}_{\mathrm{f}} \vec{f}_{1}$ is of the cortex. The habit may even be learnt de novo after the \& lesions have been inflicted. In some of Lashley's experiments rait trained to discriminate between different intensities of li.ght.o This discrimination habit is lost after destruction of the visual aref gen if it has been extremely well established. According, to Lashley therefore, the long training does not reduce the habit, of subcortical logels. But although the habit of brightness-discrimination is abolished b an occipital lesion, 
it may then be re-established by training. The re-established habit survives destruction of any given part of the remaining cortex. If this is the case it would therefore appear that the vicarious restitution of the habit is not due to any specific part of the remaining cortex. But if the second lesion is of sufficiently great extent, a second restoration does not occur, and Lashley is of the opinion that the vicarious restitution is brought about by the cortex and not by subcortical centres, but that the restoring mechanism is diffused throughout the hemispheres and not specifically concentrated in a given locus.

There is undoubtedly some sort of localization of function in the cerebral mechanism. Regarded rather crudely, much knowledge of that localization is probably sufficiently exact for the purposes of clinical neurology. But there remain unknown vast and-intricate questions concerning the working of the cerebral mechan 氶m. How do the functions of different parts of the cortex integrate in of the animal ? What is the mechanism wherebythe function of an injured cerebral mechanism may apparently becoms restored ? 